\title{
Taking Gene Therapy into the Clinic
}

\author{
Daniel H. Palmer, ${ }^{1}$ Ming-Jen Chen, ${ }^{1}$ and David J. Kerr ${ }^{2 *}$ \\ ${ }^{1}$ CRUK Institute for Cancer Studies, The Medical School, University of Birmingham, \\ Vincent Drive, Edgbaston, Birmingham B15 2TF, England, UK \\ ${ }^{2}$ Department of Clinical Pharmacology, University of Oxford, Radcliffe Infirmary, \\ Woodstock Road, Oxford, OX2 6HE, England, UK
}

Received 4 June 2002; accepted 19 July 2002

\begin{abstract}
Gene therapy represents a promising novel treatment strategy for colorectal cancer. Preclinical data has been encouraging and several clinical trials are underway. Many phase 1 trials have proven the safety of the reagents but have yet to demonstrate significant therapeutic benefit. Ongoing efforts are being made to improve the efficiency of gene delivery and accuracy of gene targeting with the aim of enhancing antitumor potency. It is envisaged that gene therapy will be used in combination with other therapies including surgery, chemotherapy, and radiotherapy to facilitate the improvements in cancer treatments in the future.
\end{abstract}

\section{INTRODUCTION}

Currently, 600 gene therapy clinical trial protocols have been activated in the U.S., $60 \%$ of which pertain to cancer gene therapy. Nearly 3500 patients have been treated within these protocols, of which approximately 2400 were patients with cancer [1]. Similarly in the U.K., of approximately 70 gene therapy protocols approved or under review by GTAC, 70\% relates to cancer gene therapy [2].

This increase in clinical trial activity is underpinned by the expansion of the number of therapeutic vectors in preclinical development and scientific innovation with respect to novel mechanistic approaches to tumour-cell kill. This article aims to provide an overview of the current clinical state of gene therapy, especially focusing on the trials, for colorectal cancer.

\section{IMMUNE STIMULATION}

The aim of immune stimulation is to activate a tumour-specific immune response, which may be either cell-mediated or antibody dependent, against the tumour cells. Several approaches to stimulate the key mediators of immune function have been tested in preclinical experiments and have now entered clinical trials, including the following approaches.

\section{Utilization of human leukocyte antigen (HLA) to stimulate $T$-cell response}

HLA class-I molecules are down regulated in up to $60 \%$ of colorectal cancers. Animal studies have demonstrated that the expression of foreign MHC (the analogue of HLA in humans) on tumours can induce a T celldependent antitumour immune response, not only to the foreign MHC but also to previously unrecognised tumour associated antigens [3].

On the basis of preclinical models, gene transfer of the HLA class-I molecule, HLA-B7, has been examined in clinical trials. In one trial, an allogeneic HLA-B7 plasmid in a lipid vector was administered via direct intratumoural injection to HLA-B7-negative patients with melanoma. Gene transfer rate was $93 \%$ when measured by polymerase chain reaction (PCR) and HLA-B7 protein was found in $50 \%$ of biopsied tumours by immunohistochemistry (IHC). Eight of 15 evaluated patients developed anti-HLA-B7 $\mathrm{CD}^{+}$cytotoxic T cells (CTLs), and 7 patients had tumour reduction (4 partial responses) [4]. A phase II trial reported a response rate in evaluable patients approaching 15\%, including two complete responses, demonstrating this to be a safe and active treatment against melanoma [5].

This trial has been extended to include patients with hepatic colorectal metastases, in which the vector was injected intratumourally under ultrasound guidance. Of 15 patients evaluated, 14 had detectable transgenic DNA by PCR, and HLA-B7 protein was detected by immunohistochemistry (IHC) in 63\% of biopsied lesions. A biological response was evident as induction of B7-specific CTLs in peripheral blood of 8 patients and also infiltration of $\mathrm{CD}^{+} \mathrm{T}$ cells into some tumours on IHC. However, no objective responses were seen [6]. This creates an interesting tension as to whether induction of a CTL response is sufficient to encourage further development of this immunogenetic approach, in the absence of bona fide reduction in tumour volume. The most obvious clinical test-bed for 
this sort of treatment would be as an adjuvant following resection of the primary tumour leaving a minimal residual volume. Although logical, clinical trials of this sort require thousands of patients, an enormous commitment of resource on the basis of an immune assay which may not correlate with efficacy.

\section{Utilization of cytokines to stimulate T cell response}

Cytokines play a key role in coordinating the immune response. Therefore, the insertion of genes encoding cytokines presents a potential strategy to increase the immunogenicity of tumours and overcome immune tolerance. Preclinical models have tested a range of cytokines including interleukins 2, 4, and 12 (IL-2, -4, -12), granulocyte macrophage colony-stimulating factor (GM-CSF), and interferon gamma (IFN- $\gamma)$. In general, in vivo models confirm that tumour-specific immunity can be generated by cytokine-transduced tumour cells. However, while this is often strong enough to prevent tumour formation/growth when rechallenged with new untransduced tumour cells, it is less efficient in eradicating established tumours.

\section{Interleukin 2 as an effector}

Autologous fibroblasts from 10 colorectal-cancer patients (used for their ease of growth in tissue culture and transducibility by retrovirus vectors expressing cytokines) were transduced with a retrovirus carrying the IL-2 gene and mixed with autologous irradiated tumour cells prior to subcutaneous reinjection [7]. In two of six evaluable patients, there was a successful induction of tumour-specific CTL precursors, however, no objective responses were demonstrated. Another approach has been to transfect autologous immune effector cells with the IL-2 gene. Preclinical studies have shown that cytokineinduced killer cells (CIKs non-MHC restricted cytotoxic lymphocytes) can eradicate tumours in nude mice. In a phase I study, 10 patients with a range of different malignancies were treated with autologous CIKs derived from peripheral blood mononuclear cells, PBMCs, transfected ex vivo by electroporation with an IL-2 plasmid before reinfusion intravenously. There was an increase in serum IFN- $\gamma$, GM-CSF, and TGF- $\beta$ during treatment and also an increase in the cytotoxic activity of circulating lymphocytes tested against a range of HLA-matched carcinoma cell lines. One patient with follicular B cell lymphoma achieved a complete response [8] and the associated side effect profile comprised mainly of fever and myalgia.

Two further phase I studies treating patients with a range of advanced cancers have utilized either allogeneic fibroblasts secreting IL-2, or an IL-2 DNA/lipid complex (leuvectin) delivered by direct intratumoural injection. Both approaches were well tolerated with evidence of biological activity (detection of IL-2 on tumour biopsy and tumour infiltration by $\mathrm{T}$ cells) in vivo as well as clinical objective responses in some patients (with melanoma or renal carcinoma).
In summary, cytokine gene therapy appears to be safe when mediated via a number of different vectors but despite evidence of biological activity objective responses have been rare.

\section{Vaccination against tumour-specific antigens}

Tumour-associated antigens have been identified for a range of human tumours including viral antigens (eg, HPV E6, E7), mutated oncogenes (eg, ras), and nonmutated onco-fetal proteins (eg, CEA). Since T cell epitopes to these antigens have been identified, they may serve as targets for CTLs under appropriate conditions. Molecular characterization of tumour-associated antigens and identification of their genes has allowed the development of recombinant vaccines in which a vector is used to introduce DNA encoding tumour-associated antigens into patients. Viruses (especially, poxviruses) present antigen and induce both humoral and cell-mediated responses. Therefore, copresentation of tumour-associated protein with the vaccinia vector may enhance immunogenicity and increase the possibility of tumour rejection.

Carcinoembryonic antigen (CEA) is a cell surface glycoprotein over expressed on the majority of colorectal cancer cells, and is expressed at low levels in normal colon and biliary epithelium. On the basis of differential expression levels, CEA has been selected as a potential target for immunotherapy approaches. Different methods have been utilized in clinical trials.

\section{Vaccinia vector}

Several phase I trials have now tested recombinant vaccinia vectors encoding full length CEA administered subcutaneously or intradermally at doses between $10^{7}-$ $10^{8} \mathrm{pfu}$ to patients with metastatic colorectal cancer [9]. Side effects include low-grade fever, fatigue, and inflammation at the injection site. The vaccine was able to induce a CTL response to CEA epitopes but no objective tumour responses.

A potential problem with vaccinia vectors is the generation of neutralizing antibodies, which may limit efficacy. Canarypox viruses, are not pathogenic in humans, do not replicate in human cells and may therefore be given repeatedly without neutralization by antibodies. A recombinant canarypox virus containing the human CEA gene has demonstrated antitumour efficacy in mice. In a phase I trial of this vector (Avipox), patients with advanced CEA positive tumours were treated with three monthly intramuscular injections. In seven of nine evaluated patients, CEA-specific CTL responses were induced without objective tumour shrinkage [10]. A novel approach to generate an immune response to CEA is the development of antiCEA “designer T cells." In a phase I trial, T cells from patients were transduced by retrovirus delivery of chimaeric Ig-T-Cell receptor genes to generate immune effector cells which bind specifically to CEA positive cells before reinfusion. This treatment was well-tolerated up to doses of 
$10^{11} \mathrm{~T}$ cells although no objective reductions in tumour volume were documented.

Most tumours do not express costimulatory molecules and therefore, expression of such molecules on tumours may enable presentation of tumour antigens directly to $\mathrm{T}$ cells, reducing the need for professional antigen presenting cells (APCs). Further, systemic immunity against unmodified tumour cells (distant bystander effect) may be evoked. Since T cell activation requires both a specific antigen epitope and a costimulatory signal, a canarypox vector expressing human CEA and B7.1 has been constructed. A phase I study of 18 patients with CEAexpressing adenocarcinoma showed it to be well-tolerated up to $4.5 \times 10^{8} \mathrm{pfu}$ with no autoimmune reactions. Two of thirteen patients with colorectal cancer achieved stable disease, correlating with an increase in CEA-specific precursor T cells.

Overall, immunogene therapy approaches are attractive and constitute about two thirds of the ongoing clinical trials for cancer treatment. In the main, clinical trials described here have so far failed to demonstrate clinically significant responses, despite clear biological activation in the form of antibody and cell-mediated response. The discrepancy may be due, at least in part, to the dynamic evolution of tumour antigens as a result of negative selection. However, it may also highlight the limitations of traditional clinical trial design whereby efficacy must first be demonstrated in the setting of advanced disease. This may not be appropriate for immunogene therapy, which is likely to be most effective against minimal residual disease.

\section{Mutant gene correction}

The phenotypic correction of key genetic aberrations in malignant cells has shown the potential to trigger the induction of apoptosis in a range of preclinical models. Different strategies including tumour suppressor gene correction (eg, p53) or oncogene suppression (eg, K-ras) have shown antitumoural effects in animal models of colorectal cancer. p53 gene correction delivered in adenoviral vectors is being tested in clinical trials in combination with conventional chemotherapy.

About $50 \%$ of colorectal cancers harbour p53 mutations. It has been shown that reexpression of wildtype-p53 in mutated colon cancer xenografts expressing the mutant variant can lead to inhibition of tumour growth and increased animal survival. A number of clinical trials using a replication-deficient adenoviral vector to deliver wild-type p53 to a range of human tumours have been carried out. Initial studies demonstrated the safety of direct intratumoural injection of these vectors and confirmed p53 gene expression even in the presence of an antiadenovirus immune response. A phase I study has assessed the safety and efficacy of a single dose of adenovirus-delivered p53 (SCH58500) administered via the hepatic artery to patients with hepatic colorectal metastases with the aim of maximizing tumour cell exposure and minimizing systemic exposure. Treatment was well-tolerated up to the maximum dose of $2.5 \times 10^{12}$ virus particles with toxicity comprizing flu-like symptoms and in four out of sixteen patients, a transient asymptomatic rise in liver transaminases. Of twelve patients who went on to receive intrahepatic chemotherapy with 5-fluorodeoxyuridine, eleven achieved a partial response [12]. This compares favourably to partial response rates of about $50 \%$ in historical controls.

Mutation of K-ras is common to many malignancies of the gastrointestinal tract. This provides a potential target for antisense oligonucleotide therapy. The introduction of synthetic oligonucleotides, capable of hybridization to specific complementary messenger RNAs, can block the expression of a single protein that plays a critical role in tumour growth. Preclinical studies of K-ras antisense therapy suggest this to be a safe, relatively nontoxic treatment.

\section{Virus-directed enzyme prodrug therapy}

Enzyme prodrug systems, also called suicide gene therapy or gene-directed enzyme prodrug system (GDEPT), are alternatives to systemic chemotherapy. This involves gene transfer, for example, via a viral vector (virus-directed enzyme prodrug therapy, VDEPT) to express viral, bacterial, or fungal enzymes in tumour cells. The enzyme can convert an inactive prodrug into a toxic metabolite, leading to tumour cell death. Compared to systemic chemotherapy, the merit of this approach is of confining generation of a short-lived cytotoxic species to the tumour, reducing systemic metabolite concentrations, and therefore limiting the potential for toxicity to normal cells, such as bone marrow and gastrointestinal tract. The main obstacle of this method is the limited gene transfer efficiency at the tumour site by vectors currently available. However, this hurdle may be partially overcome by the bystander effect. The bystander effect refers to the observation that only a fraction of the total cancer cell population needs to be transfected by the vector to lead to significant degrees of cell kill. This may be a local effect mediated by passage of the toxic metabolite (or other apoptotic factors from dying cells) to neighbouring cells either by passive diffusion, via gap junctions or via apoptotic vesicles. Alternatively, there may be an immune-mediated response that could induce a distant bystander effect. This has been observed in murine GDEPT models in which regression of distant tumour deposits is seen in immunocompetent mice but is less marked in athymic animals [13]. A logical extension of this phenomenon may be to combine GDEPT with cytokine gene therapy in order to maximize this effect.

A number of bacterial/viral enzymes have been cloned which have the capacity to catalyze the conversion of a range of prodrugs to mechanistically diverse cytotoxics. Phosphorylation of ganciclovir produces the toxic metabolite GCV-triphosphate, which competes with dGTP and inhibits DNA synthesis. This reaction 
is catalysed by herpes simplex virus (HSV)-Thymidine Kinase $(t k)$ 1000-fold more efficiently than the human nucleoside kinase, making this an attractive model for GDEPT. In a syngeneic murine model of colorectal cancer, the HSV-tk/GCV system could achieve complete tumour regression when only $9 \%$ of cells express the $t k$ gene. The lipid-insoluble GCV metabolite cannot diffuse into adjacent cells suggesting that the bystander effect may be mediated by gap-junction transport or via an immune response [14]. This system has been shown to be welltolerated and has achieved clinical responses in phase I trials of brain and prostate cancer. Similar studies have been undertaken using a replication-deficient adenovirus vector to deliver RSV-tk with similar preclinical results. A phase I trial utilizing direct intratumoural injection of this vector followed by a fixed dose of GCV in patients with hepatic metastatic colorectal cancer has been undertaken. Sixteen patients were treated with escalating doses of virus up to $1 \times 10^{13}$ particles. This treatment was well-tolerated with no dose-limiting toxicity observed, confirming the safety of the adenovirus vector delivered by the intratumoural route [15].

Bacterial or fungal cytosine deaminase (CD) is able to convert the antifungal agent, 5-fluorocytosine (5-FC), into 5-fluorouracil (5-FU), one of the most effective chemotherapeutic agents for colorectal cancer. There is some evidence suggesting that the fungal CD is superior to its bacterial counterpart, and a profound bystander effect is seen in vivo. Significant tumour regression has been achieved when only $2 \%$ of cells in xenografts expressed cytosine deaminase [16]. Furthermore, the combination of the pyrimidine salvage pathway enzyme, uracil phosphoribosyl transferase (UPRT), with CD has been shown to enhance the antitumoral effect possibly by increasing the conversion of 5-FU to 5-fluoro-deoxyuracil monophosphate, thereby accelerating a rate-limiting step in conversion of 5-FU to its cytotoxic metabolites [17]. A phase I trial of a replication-deficient adenovirus carrying the $E$. coli CD gene (Ad-GVCD-10) given intratumourally followed by oral 5-FC to patients with hepatic metastatic colorectal cancer is underway. Dose escalation to a maximum of $2 \times 10^{9} \mathrm{pfu}$ is planned. The trial comprises two arms, one of which is treated with the vector and the prodrug only, in the other, the tumour is removed after treatment so that histological and molecular analysis can be undertaken.

Nitroreductase can convert the prodrug CB1954 to a highly toxic bifunctional alkylating agent, which can cause interstrand DNA crosslinks, leading to cell death. This effect is cell cycle independent. In cell-mixing experiments, a significant bystander effect was seen when only $10 \%$ of pancreatic cancer cells expressing nitroreductase were treated with CB1954 [18]. A phase I dose-escalating study of the prodrug, CB1954 has already been completed, establishing the dose of CB1954 that can be delivered safely by the intravenous (IV) and intraperitoneal (IP) routes. Pharmacokinetic analysis showed that venous levels sufficient for clinically significant prodrug activation

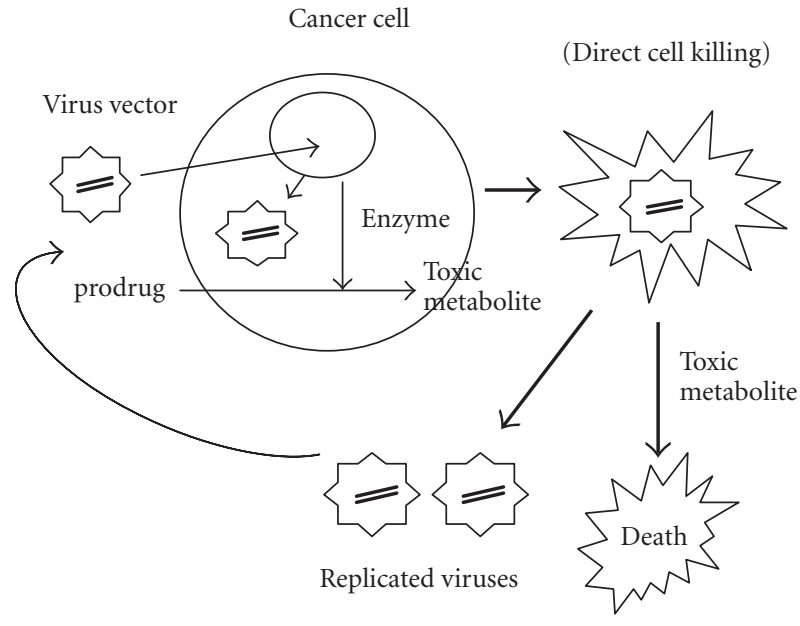

(Bystander effect)

FIGURE 1. The use of replication-competent viral vector as a carrier for therapeutic gene delivery. The potential advantages are: (1) the dual killing effect caused by viral oncolysis and the therapeutic gene system, for example, enzyme prodrug system; (2) the continuing infection by the replicated viruses to surrounding tumor cells which were not infected initially.

could be achieved, based on data from preclinical models with IP concentrations of around $100 \mu \mathrm{mol}$ [19]. Meanwhile, in the other arm of this ongoing phase I study, an E1- and E3-deleted adenovirus containing the nitroreductase gene, under the control of a cytomegalovirus promoter, is given to patients with hepatic colorectal metastasis or hepatocellular carcinoma by ultrasound-guided intratumoral injection. CB1954 will be given intravenously 48 hours after viral injection, once an adequate level of nitroreductase gene expression has been detected in resected hepatic tumours from operable patients. So far, preliminary data from this trial have demonstrated the safety of intratumoural vector administration up to $5 \times 10^{11}$ virus particles. Immunohistochemical analysis of resected tumours has confirmed nitroreductase expression which increases in a dose-dependent manner. Generally, the viral vectors used in these trials were delivered by intratumoral injection directly to the tumour site. This approach secures accurate tumour targeting but may limit the use to solitary tumours rather than to systemic disease. Clinical efficacy has not yet been shown.

A further level of specificity for these systems is the insertion of a tumour specific promotor, for example, the CEA tumour promoter, to regulate gene expression so that even if normal cells were infected, the enzyme would not be transcribed. It is envisaged that this would allow regional administration of the vector to the liver in patients undergoing resection of a primary colorectal tumour. This would be followed by systemic administration of prodrug. Given the favourable growth kinetics of microscopic metastases following resection of the primary tumour, GDEPT may play a useful role in this 
adjuvant setting. A further extension of the targeted approach would be to consider intrahepatic arterial (IHA) administration of the vector. The liver is a common site of metastasis for many cancer types and, once established, draw their blood supply from the hepatic artery. It is possible to cannulate the artery and implant a catheter which allows repeated administration of virus and/or drug, generating high local concentrations of the relevant therapeutic agent and perhaps increasing the opportunity for transfection and prodrug activation. In the adjuvant setting, where micrometastases are more likely to be served by the portal vein, it is possible to deliver the VDEPT components by an intraportal catheter inserted at the time of resection of the primary colorectal cancer.

\section{Oncolytic virus therapy}

In recent years, genetically modified oncolytic viruses including adenovirus, herpes simplex virus, and reovirus have been developed and tested. These viruses require key tumourigenic pathways to be mutated for viral replication and hence can selectively replicate in and lyse tumour cells while sparing normal cells [20]. Among them, mutants of adenovirus and herpes simplex viruses are already in trials for various cancers.

The development of replication-competent viral vectors may have the potential to improve the relatively low levels of transgene expression in many gene therapy protocols. It is envisaged that conditionally replicating vectors may be able to overcome these hurdles and, further, may have the potential to reach disseminated metastases.

\section{Oncolytic adenovirus}

The wild-type adenovirus was first used in the treatment of cervical cancer. Recently, an E1B-attenuated adenovirus, dl1520 (ONYX-015) has been tested on more than 200 patients with head and neck cancer, hepatic colorectal metastases, ovarian cancer, and pancreatic cancer. This mutant virus was engineered not to express the E1B$55-\mathrm{kDa}$ virus protein and, therefore, was initially reported to replicate specifically in cancer cells lacking functional p53, leading to cell lysis. However, it was subsequently found that this virus could also replicate efficiently in several tumour cell lines with wild-type p53. These contradictory results raised the doubt about the specificity of dl1520-mediated killing effect in p53-mutated cells. Nevertheless, recent data has shown that the loss of p14mediated Mdm2 inhibition plays an important role in supporting the replication of this virus in tumour cells with wild-type p53.

In a phase I/II trial, ONYX-015 was administered via hepatic artery infusion for patients with metastatic hepatic tumours. Two courses of a five-day infusion of 5FU/folinic acid were given concurrently. The virus was well-tolerated at the dose of $10^{11} \mathrm{pfu}$ /infusion without dose-limiting toxicities, although most of the patients developed grade-I/II fever and a few patients developed rigors after viral injection. Preliminary data showed partial responses in two of the four evaluable patients, which is comparable to the standard chemotherapeutic treatment [21].

Another trial using the same virus administered via hepatic artery infusion, intravenous infusion, or intratumoral injection, without chemotherapy, was conducted in 16 patients with primary or metastatic hepatic tumour (mainly from colorectal primaries). Tumour necrosis after viral injection was seen on CT scanning and histological analysis in all patients. No severe side effects were observed at a dose of $3 \times 10^{11} \mathrm{pfu}$ [22].

In summary, ONYX-015 has been investigated in several clinical trials, treating a range of tumours. Doses up to $2 \times 10^{12}$ virus particles have been well-tolerated when administered intratumourally, via the hepatic artery, intraperitoneally, and intravenously. No dose-limiting toxicity has been observed. Virus replication has been observed after administration by all routes, but to a variable extent depending on tumour type. There is also a confirmation that distant tumours can be infected following systemic delivery. Interestingly, ONYX-015 has demonstrated very little efficacy as a single agent in head and neck cancer (0-14\% objective response rate). However, clinical benefit has been seen where combined with chemotherapeutic agents. ONYX-015, in combination with chemotherapy (5-FU and cisplatin), has also shown promising results in a trial of head and neck tumours, with a complete response rate of $27 \%$ and a partial response rate of 36\% [23]. The combination of oncolytic virus with chemotherapeutic agents seemed synergistic but randomised, properly, powered trials comparing chemotherapy versus chemotherapy plus virus are required.

The original concept that genetically engineered viruses could specifically target tumour cells derived from the observation that deletion of $t k$ gene from HSV allowed viral replication exclusively in mitotic cells. The current oncolytic HSV's are engineered with mutations in one or both of two other genes; viral ribonucleotide reductase (ICP6), the loss of which restricts lytic virus replication to the dividing cells that retain sufficient ribonucleotide reductase activity to support the replication of the virus; and viral ICP34.4, mutation of which allows continued protein synthesis by blocking the shutdown of host cell protein synthesis normally associated with HSV infection thereby enhancing the generation of virus progeny. Such attenuated HSV's have been engineered and exploited in cancer trials for brain tumours and prostate cancer [24].

Replicating viruses have the potential to infect a greater proportion of tumour cells and to increase transgene expression. It is attractive, therefore, to combine these viruses with gene therapy strategies such as prodrug activation or cytokine expression by inserting the appropriate cDNA into the vector (Figure 1). Preclinical studies using the combination of a replication-competent HSV vector encoding the $t k$ gene have been performed. In a colorectal cancer model, the addition of GCV actually 
reduced the cytotoxic effect of the oncolytic vector, probably because of the antiviral activity of the activated prodrug. More encouraging results have been reported with E1B-attenuated adenovirus vectors encoding a combination of CD and $t k$. Data showed that the vector alone was cytotoxic, but the addition of both 5-FU and GCV further enhanced cell killing [25]. Interesting data regarding a potentially synergistic interaction between oncolytic viruses and radiotherapy is also emerging, with evidence that radiotherapy may enhance viral replication within tumours [25].

Overall, progress is being made in the clinical development of oncolytic viruses. Future clinical studies will address the optimum combination of replicating vector, gene insert, chemotherapy, and radiotherapy to maximize their therapeutic potential.

\section{FUTURE DEVELOPMENTS}

Gene therapy is now no longer in its basic scientific or clinical infancy. It is a toddler, and the initial expressions and gasps of delight that surrounded its conceptual birth have somewhat given way to the recognition that there will now be a longer haul through school days to adolescence before we can see, through properly powered randomized clinical trials, its true worth. In the meantime, several challenges remain.

(i) Improving the efficiency of gene transfer:

The recent development of replication-competent vectors for cancer gene therapy may improve gene transfer efficiency.

(ii) Improving the potency of antitumor effect:

Approaches to improve the potency of antitumor efficacy include, combination of gene therapy with conventional chemotherapy, inclusion of different enzyme activating genes within a single vector, and utilization of cytotoxic and immune effector systems.

(iii) Specific gene expression at tumour sites to avoid toxicity to normal tissues:

To limit specific gene expression to the site of tumours, tumour- or tissue-specific promoters such as CEA, Prostate Specific Antigen, and Alphafetoprotein can be used or retargeted viruses incorporating novel ligands in cell binding domains.

(iv) Safety of viral vectors:

Safety is still a concern, especially when viral vectors are used. For herpes simplex virus, the infection of this virus can be eradicated by antiviral drug (acyclovir or ganciclovir). However, for adenovirus, viral clearance is dependent on the immune system. Therefore, screening should rule out the immunocompromised patients from adenovirusmediated gene transfer protocols. Safety can be potentially improved by the development of gutless virus, chimaeric virus, minivirus, or complementary oncolytic virus.

(v) Noninvasive monitoring of transgene expression:

It is critical that noninvasive imaging systems are developed, that can detect transgene expression in vivo and evaluate the pharmacokinetics of genetic medicines to allow thorough pharmacological studies.

It is likely that gene therapies will be integrated with existing treatment modalities. The envisaged future practice for cancer treatment may involve a multimodality approach, integrating curative or debulking resection, followed by adjuvant therapies including concurrent or sequential gene therapy, chemotherapy, and radiotherapy.

\section{REFERENCES}

[1] Journal Gene Medicine website. http://www.wiley. co.uk/wileychi/genmed.

[2] Gene Therapy Advisory Committee website. http:// www.doh.gov.uk/genetics/gtac/.

[3] Ostrand-Rosenberg S, Thakur A, Clements V. Rejection of mouse sarcoma cells after transfection of MHC class II genes. J Immunol. 1990;144(10):40684071.

[4] Nabel GJ, Nabel EG, Yang ZY, et al. Direct gene transfer with DNA-liposome complexes in melanoma: expression, biologic activity, and lack of toxicity in humans. Proc Natl Acad Sci USA. 1993;90(23):1130711311.

[5] Gonzalez R, Atkins M, Schwarzenberger P, et al. Phase II trial of HLA-B7 plasmid DNA/lipid (Allovectin-7囚) immunotherapy in patients with metastatic melanoma. Proc Am Soc Clin Oncol. 2001;20(1007):252a.

[6] Rubin J, Galanis E, Pitot HC, et al. Phase I study of immunotherapy of hepatic metastases of colorectal carcinoma by direct gene transfer of an allogeneic histocompatibility antigen, HLA-B7. Gene Ther. 1997;4(5):419-425.

[7] Sobol RE, Shawler DL, Carson C, et al. Interleukin 2 gene therapy of colorectal carcinoma with autologous irradiated tumor cells and genetically engineered fibroblasts: a phase I study. Clin Cancer Res. 1999;5(9):2359-2365.

[8] Schmidt-Wolf IG, Finke S, Trojaneck B, et al. Phase I clinical study applying autologous immunological effector cells transfected with the interleukin2 gene in patients with metastatic renal cancer, colorectal cancer and lymphoma. $\mathrm{Br} J$ Cancer. 1999;81(6):1009-1016.

[9] Conry RM, Khazaeli MB, Saleh MN, et al. Phase I trial of a recombinant vaccinia virus encoding 
carcinoembryonic antigen in metastatic adenocarcinoma: comparison of intradermal versus subcutaneous administration. Clin Cancer Res. 1999; 5(9):2330-2337.

[10] Zhu MZ, Marshall J, Cole D, Schlom J, Tsang KY. Specific cytolytic T-cell responses to human CEA from patients immunized with recombinant avipoxCEA vaccine. Clin Cancer Res. 2000;6(1):24-33.

[11] Horig H, Lee DS, Conkright W, et al. Phase I clinical trial of a recombinant canarypoxvirus (ALVAC) vaccine expressing human carcinoembryonic antigen and the B7.1 co-stimulatory molecule. Cancer Immunol Immunother. 2000;49(9):504-514.

[12] Venook AP, Bergsland EK, Ring E, et al. Gene therapy of colorectal liver metastasis using recombinant adenovirus encoding wt p53 (SCH 58500) via hepatic artery infusion: a phase I study. Proc Am Soc Clin Oncol. 1998;17(1661):431a.

[13] Kuriyama S, Kikukawa M, Masui K, et al. Cytosine deaminase/5-fluorocytosine gene therapy can induce efficient anti-tumor effects and protective immunity in immunocompetent mice but not in athymic nude mice. Int J Cancer. 1999;81(4):592597.

[14] Link CJ Jr, Levy JP, McCann LZ, Moorman DW. Gene therapy for colon cancer with the herpes simplex thymidine kinase gene. J Surg Oncol. 1997; 64(4):289-294.

[15] Sung MW, Yeh HC, Thung SN, et al. Intratumoral adenovirus-mediated suicide gene transfer for hepatic metastases from colorectal adenocarcinoma: results of a phase I clinical trial. Mol Ther. 2001;4(3):182-191.

[16] Huber BE, Austin EA, Richards CA, Davis ST, Good SS. Metabolism of 5-fluorocytosine to 5-fluorouracil in human colorectal tumor cells transduced with the cytosine deaminase gene: significant antitumor effects when only a small percentage of tumor cells express cytosine deaminase. Proc Natl Acad Sci USA. 1994;91(17):8302-8306.

[17] Chung-Faye GA, Chen MJ, Green NK, et al. In vivo gene therapy for colon cancer using adenovirusmediated, transfer of the fusion gene cytosine deaminase and uracil phosphoribosyltransferase. Gene Ther. 2001;8(20):1547-1554.

[18] Green NK, Youngs DJ, Neoptolemos JP, et al. Sensitization of colorectal and pancreatic cancer cell lines to the prodrug 5-(aziridin-1-yl)-2,4dinitrobenzamide (CB1954) by retroviral transduction and expression of the E. coli nitroreductase gene. Cancer Gene Ther. 1997;4(4):229-238.

[19] Chung-Faye G, Palmer D, Anderson D, et al. Virusdirected, enzyme prodrug therapy with nitroimidazole reductase: a phase I and pharmacokinetic study of its prodrug, CB1954. Clin Cancer Res. 2001; 7(9):2662-2668.

[20] Smith ER, Chiocca EA. Oncolytic viruses as novel anticancer agents: turning one scourge against another. Expert Opin Investig Drugs. 2000;9(2):311327.

[21] Reid T, Galanis E, Abbruzzese J, et al. Intraarterial administration of a replication-selective adenovirus (dl1520) in patients with colorectal carcinoma metastatic to the liver: a phase I trial. Gene Ther. 2001;8(21):1618-1626.

[22] Habib NA, Sarraf CE, Mitry RR, et al. E1B-deleted adenovirus (dl1520) gene therapy for patients with primary and secondary liver tumors. Hum Gene Ther. 2001;12(3):219-226.

[23] Khuri FR, Nemunaitis J, Ganly I, et al. A controlled trial of intratumoral ONYX-015, a selectivelyreplicating adenovirus, in combination with cisplatin and 5-fluorouracil in patients with recurrent head and neck cancer. Nat Med. 2000;6(8):879-885.

[24] Walker JR, McGeagh KG, Sundaresan P, Jorgensen TJ, Rabkin SD, Martuza RL. Local and systemic therapy of human prostate adenocarcinoma with the conditionally replicating herpes simplex virus vector G207. Hum Gene Ther. 1999;10(13):2237-2243.

[25] Rogulski KR, Wing MS, Paielli DL, Gilbert JD, Kim JH, Freytag SO. Double suicide gene therapy augments the antitumor activity of a replicationcompetent lytic adenovirus through enhanced cytotoxicity and radiosensitization. Hum Gene Ther. 2000;11(1):67-76.

\footnotetext{
* Corresponding author. E-mail: david.kerr@clinpharm.ox.ac.uk Fax: +44 1865 224538; Tel: +44 1865224482
} 

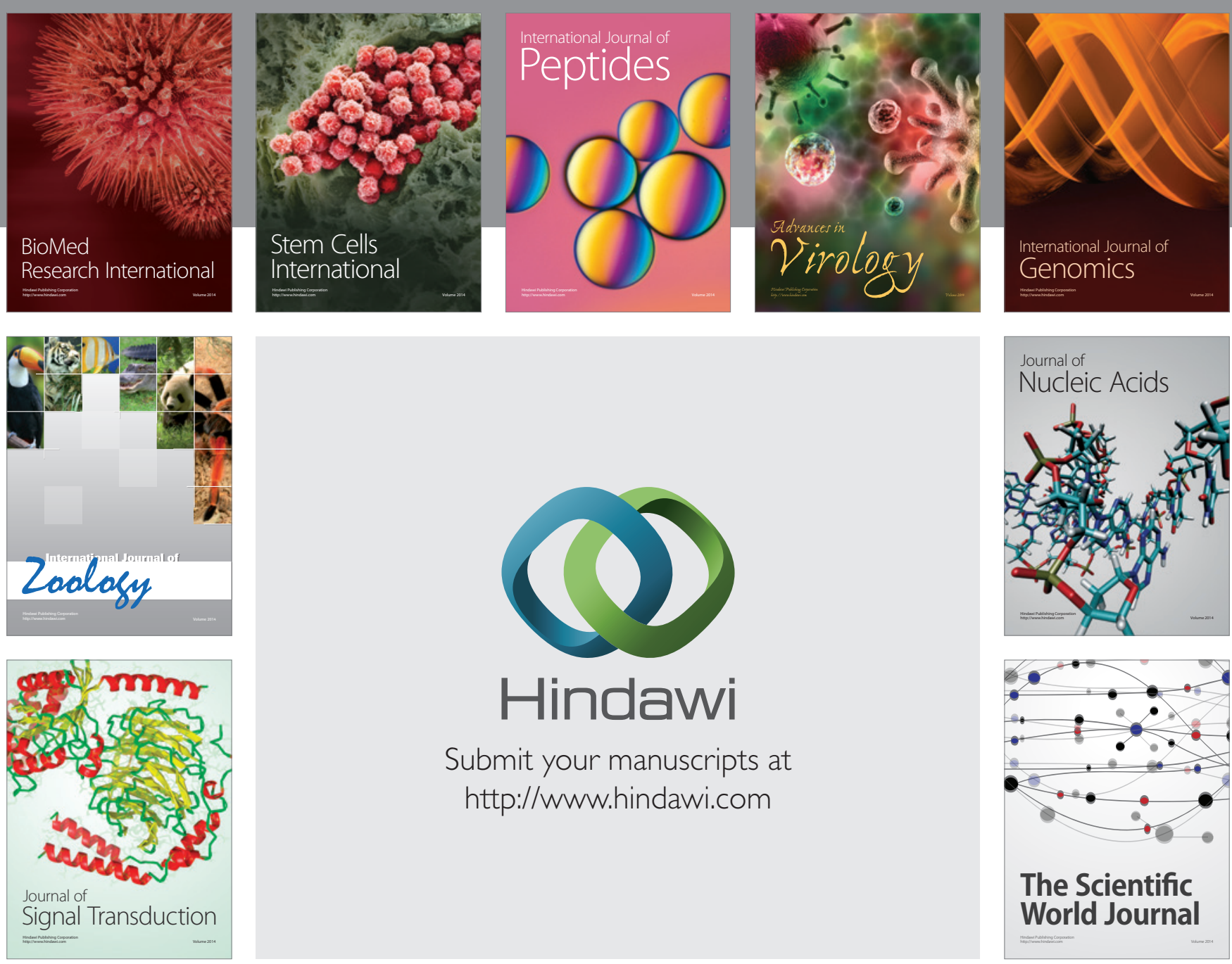

Submit your manuscripts at

http://www.hindawi.com
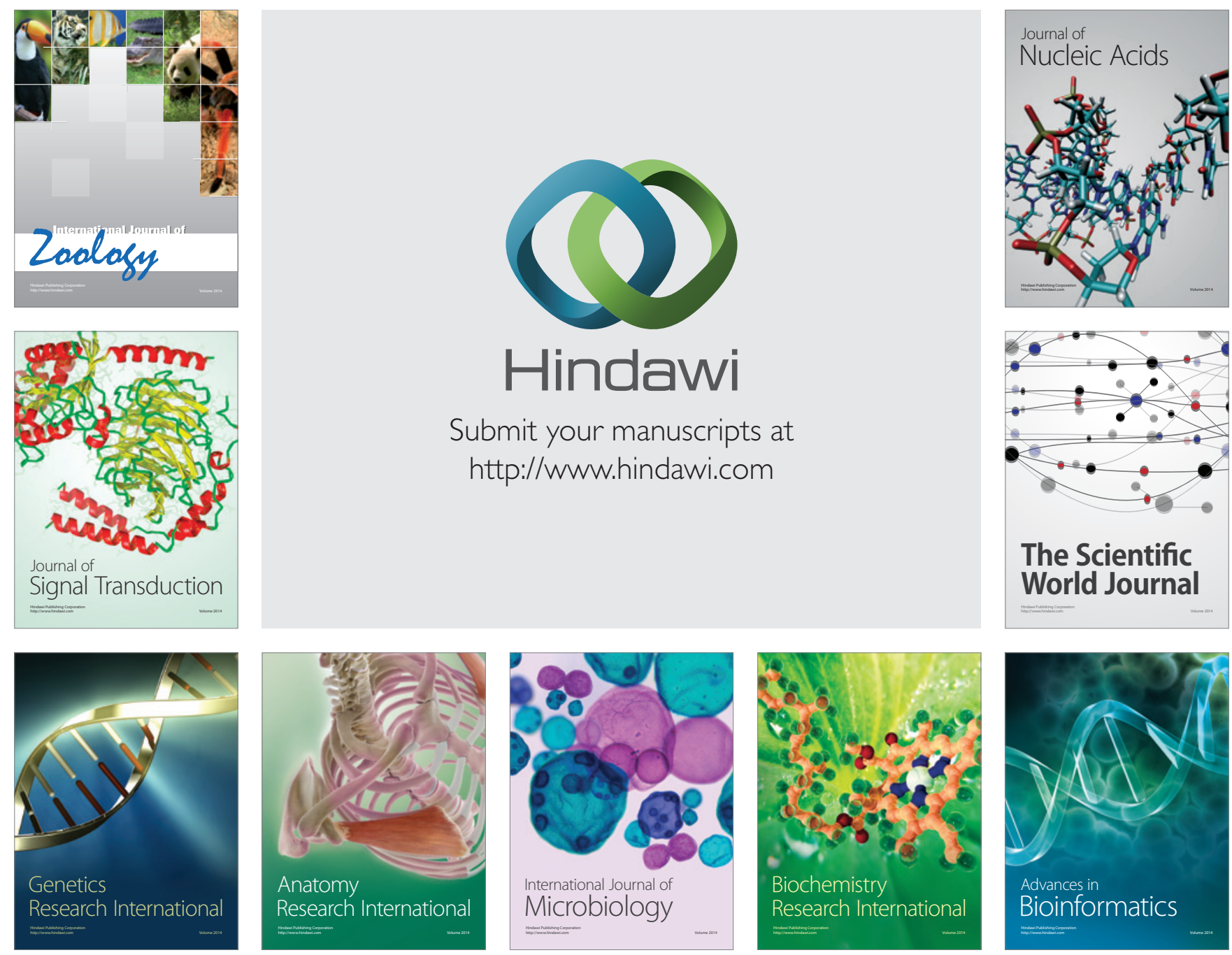

The Scientific World Journal
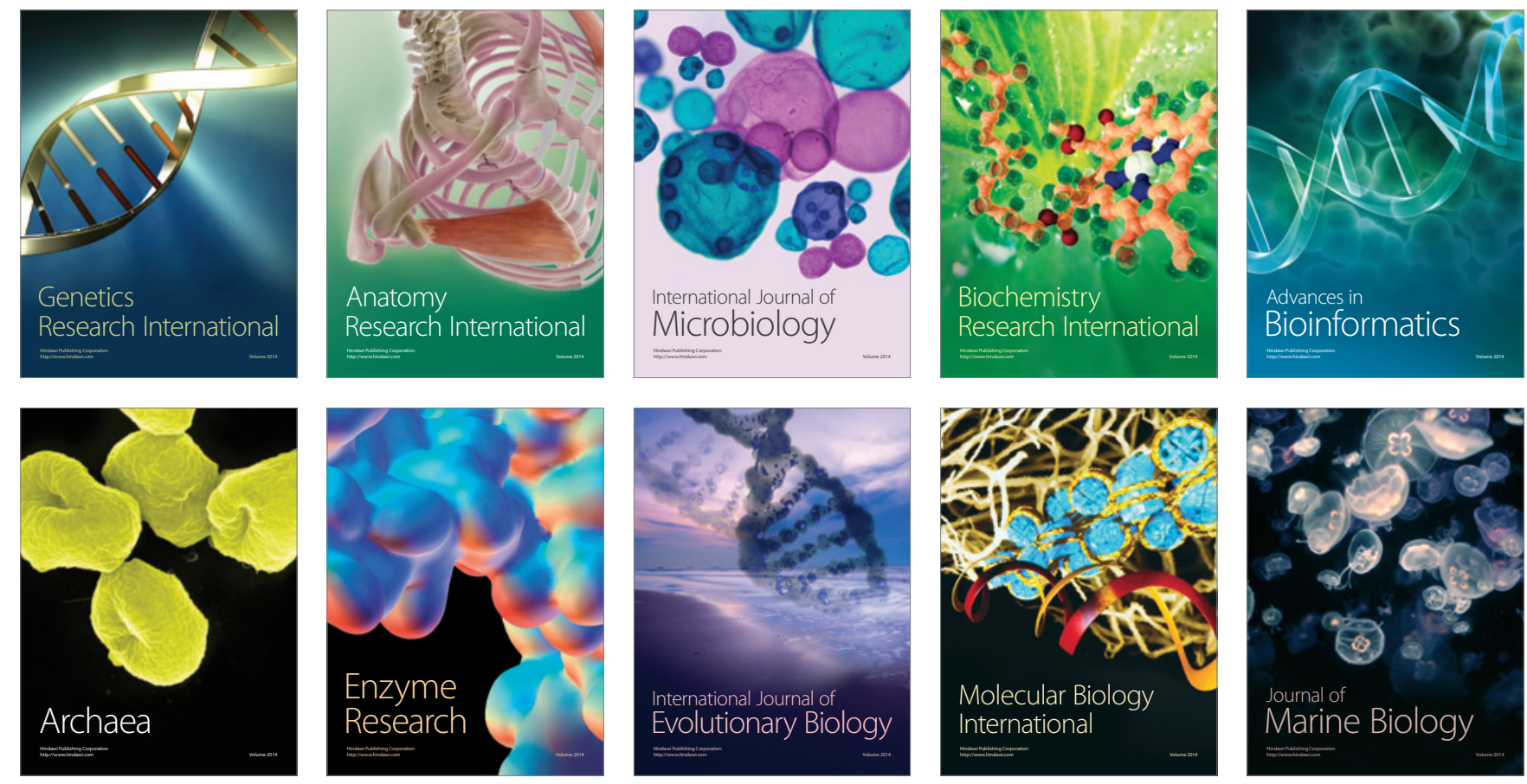